\title{
Quantum theory of light diffraction
}

\author{
Xiang-Yao $\mathrm{Wu}^{a}{ }^{*}$, Bai-Jun Zhang ${ }^{a}$, Jing-Hai Yang ${ }^{a}$, Li-Xin Chi ${ }^{a}$, Xiao-Jing Liu ${ }^{a}$ \\ Yi-Heng $\mathrm{Wu}^{a}$, Qing-Cai Wang ${ }^{a}$, Yan Wang ${ }^{a}$, Jing-Wu $\mathrm{Li}^{b}$ and Yi-Qing Guo ${ }^{c}$ \\ a.Institute of Physics, Jilin Normal University, Siping 136000, China \\ b. Institute of Physics, Xuzhou Normal University, Xuzhou 221000, China \\ c. Institute of High Energy Physics, P. O. Box 918(3), Beijing 100049, China
}

\begin{abstract}
At present, the theory of light diffraction only has the simple wave-optical approach. In this paper, we study light diffraction with the approach of relativistic quantum theory. We find that the slit length, slit width, slit thickness and wave-length of light have affected to the diffraction intensity and form of diffraction pattern. However, the effect of slit thickness on the diffraction pattern can not be explained by wave-optical approach, and it can be explained in quantum theory. We compare the theoretical results with single and multiple slits experiment data, and find the theoretical results are accordance with the experiment data. Otherwise, we give some theory prediction. We think all the new prediction will be tested by the light diffraction experiment.
\end{abstract}

PACS numbers: 03.65.-w 42.25.Fx

Keywords: Quantum theory; Light diffraction

\section{Introduction}

It is known that the nonclassical phenomena of two photon interference [1] and two- photon ghost diffraction and imaging [2], [3] have classical counterparts. Two photon interference of classical light has been first discovered in the pioneering experiments by Hanbury Brown and Twiss [4] and since then was observed with various sources, including pseudothermal ones [5], true thermal ones [6], and coherent ones [7]. Somewhat later, ghost imaging with classical light has been demonstrated, both in the near-field and far-field domains [8], [9], [10]. The present optical imaging technologies, such as optical lithography, have reached a spatial resolution in the sub-micrometer range, which comes up against the diffraction limit due to the wavelength of light. However, the guiding principle of such technology is still based on the classical diffraction theory established by Fresnel, Kirchhoff and others more than a hundred years ago. Recently, the use of quantumcorrelated photon pairs (biphotons) to overcome the classical diffraction limit was proposed and attracted much attention. Obviously, quantum theory approaches are necessary to explain the diffraction-interference of the quantum-correlated multi photon state. As is well known, the classical optics with its standard wavetheoretical methods and approximations, such as Huygens' and Kirchhoff's theory, has been successfully applied to classical optics, and has yielded good agreement with many experiments. However, light interference and diffraction are quantum phenomena, and its full description needs quantum theory approach. In 1924, Epstein and Ehrenfest had firstly studied light diffraction with the old quantum theory, i.e., the quantum mechanics of correspondence principle, and obtained a identical result with the classical optics [11]. In this work, we study the single-slit and multiple-slit diffraction of light with the approach of relativistic quantum theory of photon. In view of quantum theory, the light has the nature of wave, and the wave is described by wave function. As the wave equation which we study has the character of vector, we choose wave function $\vec{\psi}(\vec{r}, t)$ to describe the wave. The wave function $\vec{\psi}(\vec{r}, t)$ can be calculated with relativistic wave equation and it also has statistical meaning, i.e., $|\vec{\psi}(\vec{r}, t)|^{2}$ can be explained as the photon's probability density at the definite position. In light diffraction, because the diffraction intensity $I$ is directly proportional to $|\vec{\psi}(\vec{r}, t)|^{2}$, we can obtain the diffraction intensity by calculating the light wave function $\vec{\psi}(\vec{r}, t)$ distributing on display screen, and the light wave functions can be divided into three areas. The first area is the incident area, where the photon wave function is a plane wave. The second area is the slit area, where the light wave function can be calculated by quantum wave equation of light. The third area is the diffraction area,

* E-mail: wuxy2066@163.com 


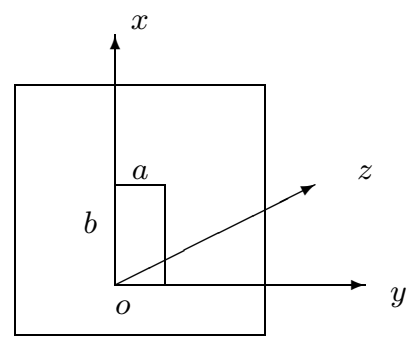

FIG. 1: The single-slit geometry, $a$ is the width and $b$ is the length of the slit.

where the light wave function can be calculated by the Kirchhoff's law. For multiple-slit diffraction, we can obtain the total diffraction wave function by superposition the diffraction wave function of every slit. In the following, we will calculate these wave functions.

The paper is organized as follows. In section 2 we calculate the light wave function in the single-slit with quantum theory approach. In section 3 we calculate the light wave function in diffraction area with the Kirchhoff's law. Section 4 is multiple-slit diffraction wave function. Section 5 is numerical result. Section 6 is a summary of results and conclusion.

\section{Quantum approach of light single-slit diffraction}

In an infinite plane, we consider a single-slit, its width $a$ and length $b$ are shown in FIG. 1 . The $x$ axis is along the slit length and the axis is along the slit width $a$. In the following, we calculate the light wave function in the single-slit with relativistic wave equation. At time $t$, we suppose that the incident plane wave travels along the $z$ axis. It is

$$
\begin{aligned}
\vec{\psi}_{0}(z, t) & =\vec{A} e^{\frac{i}{\hbar}(p z-E t)} \\
& =\sum_{j} A_{j} \cdot e^{\frac{i}{\hbar}(p z-E t)} \vec{e}_{j} \\
& =\sum_{j} \psi_{0 j} \cdot e^{-\frac{i}{\hbar} E t} \vec{e}_{j}
\end{aligned}
$$

where $\psi_{0 j}=A_{j} \cdot e^{\frac{i}{\hbar} p z}, j=x, y, z$ and $\vec{A}$ is a constant vector. The time-dependent relativistic wave equation of light is $[12]$

$$
i \hbar \frac{\partial}{\partial t} \vec{\psi}(\vec{r}, t)=c \hbar \nabla \times \vec{\psi}(\vec{r}, t)+V \vec{\psi}(\vec{r}, t)
$$

where $c$ is light velocity. From Eq. (2), we can find the light wave function $\vec{\psi}(\vec{r}, t) \rightarrow 0$ when $V(\vec{r}) \rightarrow \infty$. The potential energy of light in the single-slit is

$$
\begin{array}{rlrl}
V(x, y, z) & =0 & 0 \leq x \leq b, 0 \leq y \leq a, 0 \leq z \leq c^{\prime} \\
& =\infty \quad \text { otherwise, }
\end{array}
$$

where $c^{\prime}$ is the slit thickness. We can get the time-dependent relativistic wave equation in the slit $(V(x, y, z)=$ $0)$, it is

$$
i \hbar \frac{\partial}{\partial t} \vec{\psi}(\vec{r}, t)=c \hbar \nabla \times \vec{\psi}(\vec{r}, t)
$$

by derivation on Eq. (4) about the time t and multiplying $i \hbar$ both sides, we have

$$
(i \hbar)^{2} \frac{\partial^{2}}{\partial t^{2}} \vec{\psi}(\vec{r}, t)=c \hbar \nabla \times i \hbar \frac{\partial}{\partial t} \vec{\psi}(\vec{r}, t),
$$


substituting Eq. (4) into (5), we have

$$
\frac{\partial^{2}}{\partial t^{2}} \vec{\psi}(\vec{r}, t)=-c^{2}\left[\nabla(\nabla \cdot \vec{\psi}(\vec{r}, t))-\nabla^{2} \vec{\psi}(\vec{r}, t)\right],
$$

where the formula $\nabla \times \nabla \times \vec{B}=\nabla(\nabla \cdot \vec{B})-\nabla^{2} \vec{B}$. From Ref. [11], the photon wave function is $\vec{\psi}(\vec{r}, t)=$ $\sqrt{\frac{\varepsilon_{0}}{2}}(\vec{E}(\vec{r}, t)+i \sigma c \vec{B}(\vec{r}, t))$, we have

$$
\nabla \cdot \vec{\psi}(\vec{r}, t)=0
$$

from Eq. (6) and (7), we have

$$
\left(\frac{\partial^{2}}{\partial t^{2}}-c^{2} \nabla^{2}\right) \vec{\psi}(\vec{r}, t)=0
$$

The Eq. (8) is the same as the classical wave equation of light. Here, it is a quantum wave equation of light, since it is obtained from the relativistic wave equation (2), and it satisfied the new quantum boundary condition: when $\vec{\psi}(\vec{r}, t) \rightarrow 0, V(\vec{r}) \rightarrow \infty$. It is different from the classic boundary condition.

When the photon wave function $\vec{\psi}(\vec{r}, t)$ change with determinate frequency $\omega$, the wave function of photon can be written as

$$
\vec{\psi}(\vec{r}, t)=\vec{\psi}(\vec{r}) e^{-i \omega t}
$$

substituting Eq. (9) into (8), we can get

$$
\frac{\partial^{2} \vec{\psi}(\vec{r})}{\partial x^{2}}+\frac{\partial^{2} \vec{\psi}(\vec{r})}{\partial y^{2}}+\frac{\partial^{2} \vec{\psi}(\vec{r})}{\partial z^{2}}+\frac{4 \pi^{2}}{\lambda^{2}} \vec{\psi}(\vec{r})=0
$$

and the wave function satisfies boundary conditions

$$
\begin{aligned}
\vec{\psi}(0, y, z) & =\vec{\psi}(b, y, z)=0, \\
\vec{\psi}(x, 0, z) & =\vec{\psi}(x, a, z)=0 .
\end{aligned}
$$

The photon wave function $\vec{\psi}(\vec{r})$ can be wrote

$$
\begin{aligned}
\vec{\psi}(\vec{r}) & =\psi_{x}(\vec{r}) \vec{e}_{x}+\psi_{y}(\vec{r}) \vec{e}_{y}+\psi_{z}(\vec{r}) \vec{e}_{z} \\
& =\sum_{j=x, y, z} \psi_{j}(\vec{r}) \vec{e}_{j},
\end{aligned}
$$

where $j$ is $x, y$ or $z$. Substituting Eq. (13) into (10), (11) and (12), we have the component equation

$$
\begin{gathered}
\frac{\partial^{2} \psi_{j}(\vec{r})}{\partial x^{2}}+\frac{\partial^{2} \psi_{j}(\vec{r})}{\partial y^{2}}+\frac{\partial^{2} \psi_{j}(\vec{r})}{\partial z^{2}}+\frac{4 \pi^{2}}{\lambda^{2}} \psi_{j}(\vec{r})=0 . \\
\psi_{j}(0, y, z)=\psi_{j}(b, y, z)=0 \\
\psi_{j}(x, 0, z)=\psi_{j}(x, a, z)=0 .
\end{gathered}
$$

The partial differential equation (14) can be solved by the method of separation of variable. By writing

$$
\psi_{j}(x, y, z)=X_{j}(x) Y_{j}(y) Z_{j}(z) .
$$


From Eq. (14), (15), (16) and (17), we can get the general solution of Eq. (14)

$$
\psi_{j}(x, y, z)=\sum_{m n} D_{m n j} \sin \frac{n \pi x}{b} \sin \frac{m \pi y}{a} e^{i \sqrt{\frac{4 \pi^{2}}{\lambda^{2}}-\frac{n^{2} \pi^{2}}{b^{2}}-\frac{m^{2} \pi^{2}}{a^{2}}} z},
$$

since the wave functions are continuous at $z=0$, we have

$$
\left.\vec{\psi}_{0}(x, y, z ; t)\right|_{z=0}=\left.\vec{\psi}(x, y, z ; t)\right|_{z=0},
$$

or, equivalently,

$$
\left.\psi_{0 j}(x, y, z)\right|_{z=0}=\left.\psi_{j}(x, y, z)\right|_{z=0} . \quad(j=x, y, z)
$$

From Eq. (1), (18) and (20), we obtain the coefficient $D_{m n j}$ by fourier transform

$$
\begin{array}{rlr}
D_{m n j} & =\frac{4}{a b} \int_{0}^{a} \int_{0}^{b} A_{j} \sin \frac{n \pi \xi}{b} \sin \frac{m \pi \eta}{a} d \xi d \eta \\
& =\frac{16 A_{j}}{m n \pi^{2}} & m, n, \text { odd } \\
& =0 \quad \text { otherwise }, \quad(j=x, y, z)
\end{array}
$$

substituting Eq. (21) into (18), we have

$$
\begin{aligned}
\psi_{j}(x, y, z)= & \sum_{m, n=0}^{\infty} \frac{16 A_{j}}{(2 m+1)(2 n+1) \pi^{2}} \sin \frac{(2 n+1) \pi x}{b} \sin \frac{(2 m+1) \pi y}{a} \\
& e^{i \sqrt{\frac{4 \pi^{2}}{\lambda^{2}}-\frac{(2 n+1)^{2} \pi^{2}}{b^{2}}-\frac{(2 m+1)^{2} \pi^{2}}{a^{2}}} z}, \quad(j=x, y, z)
\end{aligned}
$$

substituting Eq. (22) into (9) and (13), we can obtain the photon wave function in slit

$$
\begin{aligned}
\vec{\psi}(x, y, z ; t)= & \sum_{j=x, y, z} \psi_{j}(x, y, z, t) \vec{e}_{j} \\
= & \sum_{j=x, y, z} \sum_{m, n=0}^{\infty} \frac{16 A_{j}}{(2 m+1)(2 n+1) \pi^{2}} \sin \frac{(2 n+1) \pi x}{b} \sin \frac{(2 m+1) \pi y}{a} \\
& e^{i \sqrt{\frac{4 \pi^{2}}{\lambda^{2}}-\frac{(2 n+1)^{2} \pi^{2}}{b^{2}}-\frac{(2 m+1)^{2} \pi^{2}}{a^{2}} z}} e^{-i \omega t} \vec{e}_{j} .
\end{aligned}
$$

We can consider the case of limit, i.e., the slit length $b$ is infinity, and the Eq. (8) and (10) become

$$
\begin{gathered}
\frac{\partial^{2}}{\partial t^{2}} \vec{\psi}(y, z, t)-c^{2}\left(\frac{\partial^{2}}{\partial y^{2}}+\frac{\partial^{2}}{\partial z^{2}}\right) \vec{\psi}(y, z, t)=0, \\
\frac{\partial^{2} \vec{\psi}(y, z)}{\partial y^{2}}+\frac{\partial^{2} \vec{\psi}(y, z)}{\partial z^{2}}+\frac{4 \pi^{2}}{\lambda^{2}} \vec{\psi}(y, z)=0,
\end{gathered}
$$

we can easily obtain the light wave function in the single-slit when $b \rightarrow \infty$

$$
\begin{aligned}
\vec{\psi}(y, z ; t)= & \sum_{j=y, z} \psi_{j}(x, y, z, t) \vec{e}_{j} \\
= & \sum_{j=x, y, z} \sum_{m=0}^{\infty} \frac{4 A_{j}}{(2 m+1) \pi} \sin \frac{(2 m+1) \pi y}{a} \\
& e^{i \sqrt{\frac{4 \pi^{2}}{\lambda^{2}}-\frac{(2 m+1)^{2} \pi^{2}}{a^{2}}}} e^{-i \omega t} \vec{e}_{j} .
\end{aligned}
$$




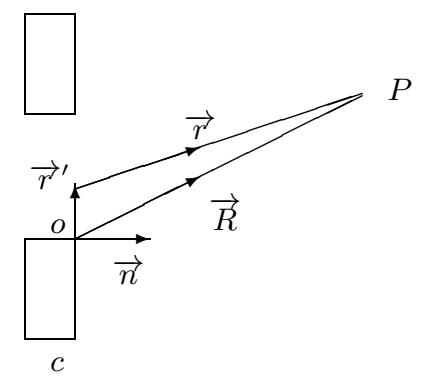

FIG. 2: The diffraction area of single-slit

\section{The wave function of light diffraction}

In the section 2 , we have calculated the photon wave function in slit. In the following, we will calculate diffraction wave function. we can calculate the wave function in the diffraction area. From the slit wave function component $\psi_{j}(\vec{r}, t)$, we can calculate its diffraction wave function component $\Phi_{j}(\vec{r}, t)$ by Kirchhoff's law. It can be calculated by the formula[13]

$$
\Phi_{j}(\vec{r}, t)=-\frac{1}{4 \pi} \int_{s_{0}} \frac{e^{i k r}}{r} \vec{n} \cdot\left[\nabla^{\prime} \psi_{j}+\left(i k-\frac{1}{r}\right) \frac{\vec{r}}{r} \psi_{j}\right] d s .
$$

the total diffraction wave function is

$$
\vec{\Phi}(\vec{r}, t)=\sum_{j=x, y, z} \Phi_{j}(\vec{r}, t) \vec{e}_{j}
$$

the diffraction area is shown in FIG. 2, where $k=\frac{2 \pi}{\lambda}$ is wave vector, $s_{0}$ is the area of the single-slit, $\vec{r}^{\prime}$ the position of a point on the surface $\left(z=c^{\prime}\right), P$ is an arbitrary point in the diffraction area, and the $\vec{n}$ is a unit vector, which is normal to the surface of the single-slit. From FIG. 2, we have

$$
\begin{aligned}
r & =R-\frac{\vec{R}}{R} \cdot \vec{r}^{\prime} \\
& \approx R-\frac{\vec{r}}{r} \cdot \vec{r}^{\prime} \\
& =R-\frac{\overrightarrow{k_{2}}}{k} \cdot \vec{r}^{\prime}
\end{aligned}
$$

then,

$$
\begin{aligned}
\frac{e^{i k r}}{r} & =\frac{e^{i k\left(R-\frac{\vec{r}}{r} \cdot \vec{r}^{\prime}\right)}}{R-\frac{\vec{r}}{r} \cdot \vec{r}^{\prime}} \\
& \approx \frac{e^{i k R} e^{-i \vec{k}_{2} \cdot \vec{r}^{\prime}}}{R} \quad\left(\left|\vec{r}^{\prime}\right| \ll R\right),
\end{aligned}
$$


where $\overrightarrow{k_{2}}=k \frac{\vec{r}}{r}$. Substituting Eq. (22), (29) and (30) into (27), one can obtain

$$
\begin{aligned}
\Phi_{j}(\vec{r}, t)= & -\frac{e^{i k R}}{4 \pi R} e^{-i \omega t} \int_{s_{0}} e^{-i \overrightarrow{k_{2}} \cdot \vec{r}^{\prime}} \sum_{m=0}^{\infty} \sum_{n=0}^{\infty} \frac{16 A_{j}}{(2 m+1)(2 n+1) \pi^{2}} \\
& e^{i \sqrt{\frac{4 \pi^{2}}{\lambda^{2}}-\left(\frac{(2 n+1) \pi}{b}\right)^{2}-\left(\frac{(2 m+1) \pi}{a}\right)^{2}} \cdot c^{\prime}} \sin \frac{(2 n+1) \pi}{b} x^{\prime} \sin \frac{(2 m+1) \pi}{a} y^{\prime} \\
& {\left[i \sqrt{\frac{4 \pi^{2}}{\lambda^{2}}-\left(\frac{(2 n+1) \pi}{b}\right)^{2}-\left(\frac{(2 m+1) \pi}{a}\right)^{2}}+i \vec{n} \cdot \overrightarrow{k_{2}}-\frac{\vec{n} \cdot \vec{R}}{R^{2}}\right] d x^{\prime} d y^{\prime} . }
\end{aligned}
$$

Assume that the angle between $\overrightarrow{k_{2}}$ and $x$ axis ( $y$ axis) is $\frac{\pi}{2}-\alpha\left(\frac{\pi}{2}-\beta\right)$, and $\alpha(\beta)$ is the angle between $\overrightarrow{k_{2}}$ and the surface of $y z(x z)$, then we have

$$
\begin{gathered}
k_{2 x}=k \sin \alpha, \quad k_{2 y}=k \sin \beta, \\
\vec{n} \cdot \overrightarrow{k_{2}}=k \cos \theta,
\end{gathered}
$$

where $\theta$ is the angle between $\overrightarrow{k_{2}}$ and $z$ axis. Substituting Eq. (32) and (33) into (31) gives

$$
\begin{aligned}
\Phi_{j}(x, y, z ; t)= & -\frac{e^{i k R}}{4 \pi R} e^{-i \omega t} \sum_{m=0}^{\infty} \sum_{n=0}^{\infty} \frac{16 A_{j}}{(2 m+1)(2 n+1) \pi^{2}} e^{i \sqrt{\frac{4 \pi^{2}}{\lambda^{2}}-\left(\frac{(2 n+1) \pi}{b}\right)^{2}-\left(\frac{(2 m+1) \pi}{a}\right)^{2}} \cdot c^{\prime}} \\
& {\left[i \sqrt{\frac{4 \pi^{2}}{\lambda^{2}}-\left(\frac{(2 n+1) \pi}{b}\right)^{2}-\left(\frac{(2 m+1) \pi}{a}\right)^{2}}+\left(i k-\frac{1}{R}\right) \sqrt{\cos ^{2} \alpha-\sin ^{2} \beta}\right] } \\
& \int_{0}^{b} e^{-i k \sin \alpha \cdot x^{\prime}} \sin \frac{(2 n+1) \pi}{b} x^{\prime} d x^{\prime} \int_{0}^{a} e^{-i k \sin \beta \cdot y^{\prime}} \sin \frac{(2 m+1) \pi}{a} y^{\prime} d y^{\prime} .
\end{aligned}
$$

Substituting Eq. (34) into (28), one can get

$$
\begin{aligned}
\vec{\Phi}(x, y, z ; t)= & -\frac{e^{i k R}}{4 \pi R} e^{-i \omega t} \sum_{j=s, y, z} \sum_{m=0}^{\infty} \sum_{n=0}^{\infty} \frac{16 A_{j}}{(2 m+1)(2 n+1) \pi^{2}} e^{i \sqrt{\frac{4 \pi^{2}}{\lambda^{2}}-\left(\frac{(2 n+1) \pi}{b}\right)^{2}-\left(\frac{(2 m+1) \pi}{a}\right)^{2}} \cdot c^{\prime}} \\
& {\left[i \sqrt{\frac{4 \pi^{2}}{\lambda^{2}}-\left(\frac{(2 n+1) \pi}{b}\right)^{2}-\left(\frac{(2 m+1) \pi}{a}\right)^{2}}+\left(i k-\frac{1}{R}\right) \sqrt{\cos ^{2} \alpha-\sin ^{2} \beta}\right] } \\
& \int_{0}^{b} e^{-i k \sin \alpha \cdot x^{\prime}} \sin \frac{(2 n+1) \pi}{b} x^{\prime} d x^{\prime} \int_{0}^{a} e^{-i k \sin \beta \cdot y^{\prime}} \sin \frac{(2 m+1) \pi}{a} y^{\prime} d y^{\prime} \vec{e}_{j} .
\end{aligned}
$$

Eq. (35) is the total diffraction wave function in the diffraction area. From the wave function, we can obtain the diffraction intensity $I$ on the display screen, we have

$$
I \propto|\vec{\Phi}(x, y, z ; t)|^{2} .
$$

\section{Multiple-slit diffraction wave function of light}

From Eq. (23), in the first slit, the photon wave function $\vec{\psi}_{1}(x, y, z ; t)$ is

$$
\begin{aligned}
\vec{\psi}_{1}(x, y, z ; t)= & \sum_{j=x, y, z} \sum_{m, n=0}^{\infty} \frac{16 A_{j}}{(2 m+1)(2 n+1) \pi^{2}} \sin \frac{(2 n+1) \pi x}{b} \sin \frac{(2 m+1) \pi y}{a} \\
& e^{i \sqrt{\frac{4 \pi^{2}}{\lambda^{2}}-\frac{(2 n+1)^{2} \pi^{2}}{b^{2}}-\frac{(2 m+1)^{2} \pi^{2}}{a^{2}}} z} e^{-i \omega t} \vec{e}_{j} .
\end{aligned}
$$




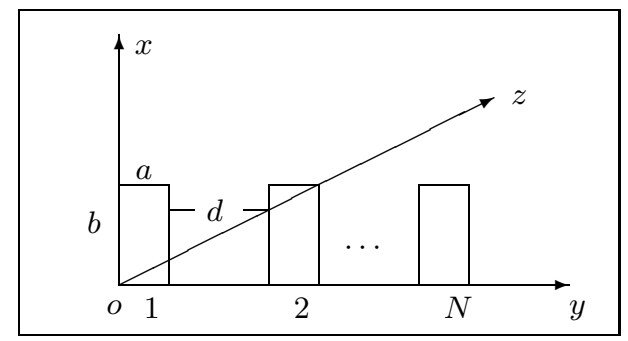

FIG. 3: Multiple-slit geometry with $a$ the single slit width, $b$ the slit length and $d$ the distance between the two slit.

From FIG. 3, in the second slit, when we make the coordinate translations :

$$
\begin{aligned}
& x^{\prime}=x \\
& y^{\prime}=y-(a+d) \\
& z^{\prime}=z,
\end{aligned}
$$

we can obtain the second slit photon wave function by the first slit photon wave function. It is

$$
\begin{aligned}
\vec{\psi}_{2}(x, y, z ; t)= & \sum_{j=x, y, z} \sum_{m, n=0}^{\infty} \frac{16 A_{j}}{(2 m+1)(2 n+1) \pi^{2}} \sin \frac{(2 n+1) \pi x}{b} \sin \frac{(2 m+1) \pi[y-(a+d)]}{a} \\
& e^{i \sqrt{\frac{4 \pi^{2}}{\lambda^{2}}-\frac{(2 n+1)^{2} \pi^{2}}{b^{2}}-\frac{(2 m+)^{2} \pi^{2}}{a^{2}}}} e^{-i \omega t} \vec{e}_{j} .
\end{aligned}
$$

Similarly, we can also obtain the N-th photon wave function. It is

$$
\begin{aligned}
\vec{\psi}_{N}(x, y, z ; t)= & \sum_{j=x, y, z} \sum_{m, n=0}^{\infty} \frac{16 A_{j}}{(2 m+1)(2 n+1) \pi^{2}} \sin \frac{(2 n+1) \pi x}{b} \sin \frac{(2 m+1) \pi[y-(N-1)(a+d)]}{a} \\
& e^{i \sqrt{\frac{4 \pi^{2}}{\lambda^{2}}-\frac{(2 n+1)^{2} \pi^{2}}{b^{2}}-\frac{(2 m+1)^{2} \pi^{2}}{a^{2}}} z} e^{-i \omega t} \vec{e}_{j} .
\end{aligned}
$$

With the Kirchhoff's law, similar as Eq. (35), we can get the light diffraction wave function in every slit, they are

$$
\begin{aligned}
\vec{\Phi}_{1}(x, y, z ; t)= & -\frac{e^{i k R}}{4 \pi R} e^{-i \omega t} \sum_{j=x, y, z} \sum_{m=0}^{\infty} \sum_{n=0}^{\infty} \frac{16 A_{j}}{(2 m+1)(2 n+1) \pi^{2}} e^{i \sqrt{\frac{4 \pi^{2}}{\lambda^{2}}-\left(\frac{(2 n+1) \pi}{b}\right)^{2}-\left(\frac{(2 m+1) \pi}{a}\right)^{2}} \cdot c^{\prime}} \\
& {\left[i \sqrt{\frac{4 \pi^{2}}{\lambda^{2}}-\left(\frac{(2 n+1) \pi}{b}\right)^{2}-\left(\frac{(2 m+1) \pi}{a}\right)^{2}}+\left(i k-\frac{1}{R}\right) \sqrt{\cos ^{2} \alpha-\sin ^{2} \beta}\right] } \\
& \int_{0}^{b} e^{-i k \sin \alpha \cdot x^{\prime}} \sin \frac{(2 n+1) \pi}{b} x^{\prime} d x^{\prime} \int_{0}^{a} e^{-i k \sin \beta \cdot y^{\prime}} \sin \frac{(2 m+1) \pi}{a} y^{\prime} d y^{\prime} \vec{e}_{j} . \\
\vec{\Phi}_{2}(x, y, z ; t)= & -\frac{e^{i k R}}{4 \pi R} e^{-i \omega t} \sum_{j=x, y, z} \sum_{m=0}^{\infty} \sum_{n=0}^{\infty} \frac{16 A_{j}}{(2 m+1)(2 n+1) \pi^{2}} e^{i \sqrt{\frac{4 \pi^{2}}{\lambda^{2}}-\left(\frac{(2 n+1) \pi}{b}\right)^{2}-\left(\frac{(2 m+1) \pi}{a}\right)^{2}} \cdot c^{\prime}} \\
& {\left[i \sqrt{\frac{4 \pi^{2}}{\lambda^{2}}-\left(\frac{(2 n+1) \pi}{b}\right)^{2}-\left(\frac{(2 m+1) \pi}{a}\right)^{2}}+\left(i k-\frac{1}{R}\right) \sqrt{\cos ^{2} \alpha-\sin ^{2} \beta}\right] } \\
& \int_{0}^{b} e^{-i k \sin \alpha \cdot x^{\prime}} \sin \frac{(2 n+1) \pi}{b} x^{\prime} d x^{\prime} \int_{a+d}^{2 a+d} e^{-i k \sin \beta \cdot y^{\prime}} \sin \frac{(2 m+1) \pi}{a} y^{\prime} d y^{\prime} \vec{e}_{j} .
\end{aligned}
$$




$$
\begin{aligned}
& \vec{\Phi}_{N}(x, y, z ; t)=-\frac{e^{i k R}}{4 \pi R} e^{-i \omega t} \sum_{j=x, y, z} \sum_{m=0}^{\infty} \sum_{n=0}^{\infty} \frac{16 A_{j}}{(2 m+1)(2 n+1) \pi^{2}} e^{i \sqrt{\frac{4 \pi^{2}}{\lambda^{2}}-\left(\frac{(2 n+1) \pi}{b}\right)^{2}-\left(\frac{(2 m+1) \pi}{a}\right)^{2}} \cdot c^{\prime}} \\
& {\left[i \sqrt{\frac{4 \pi^{2}}{\lambda^{2}}-\left(\frac{(2 n+1) \pi}{b}\right)^{2}-\left(\frac{(2 m+1) \pi}{a}\right)^{2}}+\left(i k-\frac{1}{R}\right) \sqrt{\cos ^{2} \alpha-\sin ^{2} \beta}\right]} \\
& \int_{0}^{b} e^{-i k \sin \alpha \cdot x^{\prime}} \sin \frac{(2 n+1) \pi}{b} x^{\prime} d x^{\prime} \int_{(N-1)(a+d)}^{(N-1)(a+d)+a} e^{-i k \sin \beta \cdot y^{\prime}} \sin \frac{(2 m+1) \pi}{a} y^{\prime} d y^{\prime} \vec{e}_{j} \text {. }
\end{aligned}
$$

The total diffraction wave function for the N-slit is

$$
\vec{\Phi}(x, y, z ; t)=\vec{\Phi}_{1}(x, y, z ; t)+\vec{\Phi}_{2}(x, y, z ; t)+\ldots \ldots+\vec{\Phi}_{N}(x, y, z ; t) .
$$

From Eq. (44), we can obtain the diffraction intensity $I$ on the display screen for N-slit, we have

$$
I \propto|\vec{\Phi}(x, y, z ; t)|^{2}
$$

When $b \rightarrow \infty$, we can get the light diffraction wave function in sing-slit, it is

$$
\begin{aligned}
\vec{\Phi}_{b \rightarrow \infty}(y, z ; t)= & -\frac{e^{i k R}}{4 \pi R} e^{-i \omega t} \sum_{j=x, y, z} \sum_{m=0}^{\infty} \frac{4 A_{j}}{(2 m+1) \pi} e^{i \sqrt{\frac{4 \pi^{2}}{\lambda^{2}}-\left(\frac{(2 m+1) \pi}{a}\right)^{2}} \cdot c^{\prime}} \\
& {\left[i \sqrt{\frac{4 \pi^{2}}{\lambda^{2}}-\left(\frac{(2 m+1) \pi}{a}\right)^{2}}+\left(i k-\frac{1}{R}\right) \sqrt{\left.\cos ^{2} \alpha-\sin ^{2} \beta\right]}\right.} \\
& \int_{0}^{a} e^{-i k \sin \beta \cdot y^{\prime}} \sin \frac{(2 m+1) \pi}{a} y^{\prime} d y^{\prime} \vec{e}_{j} .
\end{aligned}
$$

From Eq. (46), we can obtain the diffraction intensity $I_{b \rightarrow \infty}$ on the display screen for sing-slit when $b \rightarrow \infty$. It is

$$
I_{b \rightarrow \infty} \propto\left|\vec{\Phi}_{b \rightarrow \infty}(y, z, t)\right|^{2}
$$

In Ref. [11], the authors had firstly studied light diffraction with the old quantum theory, i.e., the quantum mechanics of the correspondence principle. They had considered a light quantum comes into three dimensional crystal lattice, and calculated the light momentum loss after collision with quantization condition and light quantum momentum formula $p=\frac{h}{\lambda}$. They obtained the relation between the deflecting angle of light quantum collision with the lattice and the lattice period. By analyzing, the authors given the expression of electronic density distributing on grating $\rho=A_{m} \sin \frac{2 \pi m x}{a}$. The coefficient $A_{m}$ could be obtained by Fourier analysis. The diffraction spectrum intensity of the $m^{t h}$ order is proportional to $A_{m}^{2}$. Finally, they had obtained the intensity formula of diffraction spectrum which was in complete agreement with the classical diffraction. The formula given a simple relation between diffraction intensity, slit width, wavelength of incident light and diffraction angle. In this paper, we present quantum theory of light diffraction using the framework of a relativistic quantum theory, and obtain the relation between diffraction intensity, slit length, slit width, slit thickness, light wavelength and diffraction angle. By calculating, we can find the theoretical results are accordance with the experiment data, and give some new theory prediction.

\section{Numerical result}

The light diffraction experiment of single and multiple slits had been reported by H.F.Neiners in 1970 [14]. In experiment [14], the optical system consists of two convex lens, the focal length $f$, a diffraction screen of slit length $b$ and slit width $a(b \gg a)$ and a display screen. The laser light source of wave length $\lambda$ 
places on the focal plane of the first convex lens, the first lens makes the light beams parallel incident on the diffraction screen, the second lens is next to the diffraction screen, and the display screen places on the focal plane of second convex lens. In the experiment, the diffraction patterns were given by photos, and not given experimental data. The author found his results can be explained excellently by the classical theoretical formula. It is

$$
I=I_{0} \frac{\sin ^{2} \beta}{\beta^{2}} \frac{\sin ^{2} N \gamma}{\sin ^{2} \gamma}
$$

where

$$
\beta=\frac{a \pi \sin \theta}{\lambda}, \quad \gamma=\frac{(a+d) \pi \sin \theta}{\lambda},
$$

$\theta$ is diffraction angle, $a$ is the width of slit, $d$ is the distance from the first slit to the second slit, and $n$ is the number of slit.

FIG. 4 (a)-(f) show the diffraction patterns from two, three, four, five, six and seven slits with $\lambda=$ $6.328 \times 10^{-7} \mathrm{~m}$, the slit width $a=0.88 \times 10^{-4} \mathrm{~m}, a+d=3.52 \times 10^{-4} \mathrm{~m}$ ( $d$ is the distance between two slits), and the distance between slit and display screen $R=4.572 \mathrm{~m}$. In FIG. 4 (a)-(f), the solid curve is our theoretical result, and the dot curve is the result of Eq. (48), i.e., the diffraction data. In FIG. 4 (a)-(f), we take the same experiment parameters in our calculation, and the theoretical input parameters are: the slit length $b=3.52 \times 10^{-4} \mathrm{~m}$, and the slit thickness $c^{\prime}=0.88 \times 10^{-4} \mathrm{~m}$. From FIG. 4 (a), we can find the calculation result is accordance with experiment data. Since the ration $\frac{a+d}{a}=4$, we find the orders $4,8,12$ $\cdots$ are missing. The conclusion is accordance with classical optics.

FIG. 4 (b) - (f) are multiple slits diffraction patterns corresponding to slit number $\mathrm{N}=3,4,5,6$, 7. We can find the calculation results are accordance with experiment data, and there are $N-2$ secondary maxima and $N-1$ minima between the two principle maxima. The conclusions are accordance with classical optics.

FIG. 5 shows the diffraction patterns for single slit. In the experiment, the light wave length $\lambda=6.328 \times$ $10^{-7} \mathrm{~m}$, the slit width $a=1.76 \times 10^{-4} \mathrm{~m}$, and the distance between slit and display screen $R=4.572 \mathrm{~m}$. In our calculation, we take the same experiment parameters above, and the theoretical input parameters are: the slit length $b=4.0 \times 10^{-4} \mathrm{~m}$, the slit thickness $c^{\prime}=1.1 \times 10^{-6} \mathrm{~m}$, and the diffraction angle $\alpha=0.001 \mathrm{rad}$. In FIG. 5, the solid curve is our theoretical result and the dot curve is the result of Eq. (48), i.e., the diffraction data. From FIG. 5, we can find the calculation result shows a good agreement with experiment data. We have compared the theoretical results with experiment data above. In the following, we give some theoretical prediction in FIG. 6 - FIG. 12. In calculation, we take the light wave length $\lambda=6.328 \times 10^{-7} \mathrm{~m}$, the distance $R=4.572 \mathrm{~m}$ and the diffraction angle $\alpha=0.001 \mathrm{rad}$.

FIG. 6 is obtained by taking the single slit width as $5 a, 10 a$ and $20 a\left(a=1.76 \times 10^{-4} \mathrm{~m}\right)$ and $b=$ $4.0 \times 10^{-4} \mathrm{~m}, c^{\prime}=1.1 \times 10^{-6} \mathrm{~m}$. From FIG. 6 , we can find when the slit width increases, the diffraction patterns become narrower, and the diffraction intensity increases.

In FIG. 7, the slit width and slit length are equal $(a=b)$, and it is obtained by taking the slit length and slit width as $\lambda, 3 \lambda$ and $5 \lambda$, and $c^{\prime}=1.1 \times 10^{-6} \mathrm{~m}$. From FIG. 7, we can find when they increase, the diffraction patterns become narrower, and the diffraction intensity increase.

In FIG. 8, we can obtain an important result, when $a=b \leq 0.1 \lambda$, the total diffraction intensity is zero, i.e., a very small hole can not produce diffraction phenomenon. It is because the incident light scatters back completely when the size of slit is very small.

FIG. 9 is obtained by taking the single slit length as $50 b, 70 b$ and infinity $\left(b=4.0 \times 10^{-4} \mathrm{~m}\right)$ and $a=$ $1.76 \times 10^{-4} \mathrm{~m}, c^{\prime}=1.1 \times 10^{-6} \mathrm{~m}$. From FIG. 9, we can obtain the following conclusions: (1) When the slit length increases, the diffraction intensity increases. (2) When the slit length changes, the width of diffraction patterns do not change.

FIG. 10 is obtained by taking the single slit thickness as $100 c^{\prime}, 1000 c^{\prime}, 2000 c^{\prime}$ and $3000 c^{\prime}\left(c^{\prime}=1.1 \times 10^{-6} \mathrm{~m}\right)$ and $a=1.76 \times 10^{-4} \mathrm{~m}, b=4.0 \times 10^{-4} \mathrm{~m}$. From FIG. 10, we can obtain the following conclusions: (1) When the slit thickness increases, the total diffraction intensity decreases. (2) When the slit thickness increases, the diffraction patterns spread over.

FIG. 11 is obtained by taking the single wave length as $10 \lambda, 20 \lambda$ and $50 \lambda\left(\lambda=6.328 \times 10^{-7} \mathrm{~m}\right)$ and $a=1.76 \times 10^{-4} \mathrm{~m}, b=4.0 \times 10^{-4} \mathrm{~m}, c^{\prime}=1.1 \times 10^{-6} \mathrm{~m}$. From FIG. 11, we can obtain the following 
conclusions: (1) When the wave length decrease, the total diffraction intensity increases and diffraction patterns become narrow. (2) When the wave length decrease, the number of diffraction patterns become more.

FIG. 12 is obtained by taking the double slit thickness as $c^{\prime}, 10 c^{\prime}$ and $50 c^{\prime}\left(c^{\prime}=0.88 \times 10^{-4} \mathrm{~m}\right)$ and $a=0.88 \times 10^{-4} \mathrm{~m}, b=4 \times 0.88 \times 10^{-4} \mathrm{~m}, a+d=3.52 \times 10^{-4} \mathrm{~m}$. From FIG. 12, we can obtain the following conclusions: (1) When the slit thickness increases, the diffraction intensity decreases. (2) In the classical optics, we know when the ratio $\frac{a+d}{a}=n(n=1,2,3 \ldots)$, the orders $n, 2 n, 3 n, \ldots$ are missing in double slit diffraction. We find when the slit thickness takes $c^{\prime}$, and the ratio $\frac{a+d}{a}=4$, the orders $4,8,12, \ldots$ are missing. (3) When the slit thickness increases, such as $10 c^{\prime}$ and $50 c^{\prime}$, we find the missing-order phenomenon disappears.

From FIG. 4 and FIG. 5, we can find our calculation results are accordance with the experiment data, the classical optic results and the forepart quantum theory results [13]. From FIG. 6 to FIG. 12, we give some new prediction, which can be tested by light diffraction experiment.

\section{Conclusion}

In conclusion, we have studied the single-slit, double-slit and multiple-slit diffraction of light with the relativistic quantum mechanical approach. We give the relation among diffraction intensity, slit length, slit width, slit thickness, wave length of light and diffraction angle. Our calculation results are accordance with the experiment data of the single-slit, double-slit and multiple-slit diffraction. Otherwise, we study the slit length, slit width, slit thickness, the wave length of light affect on the diffraction intensity and form of diffraction pattern. However, the slit length and slit thickness affect on the diffraction pattern can not be obtained in classical optic. In double slit diffraction, we find when the ratio $\frac{a+d}{a}=n(\mathrm{n}=1,2,3 \ldots)$, the orders $\mathrm{n}, 2 \mathrm{n}, 3 \mathrm{n}, \ldots$ are missing. When the slit thickness increase, the missing order phenomenon disappears. In multiple slit diffraction, we find there are N-2 secondary maxima and N-1 minima between the two principle maxima, we think that all the new prediction in our work can be tested by light diffraction experiment.

1 R. Ghosh and L. Mandel, Phys. Rev. Lett. 59, 1903 (1987).

2 D. V. Strekalov, A. V. Sergienko, D. N. Klyshko, and Y. H. Shih, Phys. Rev. Lett. 74, 3600-3603 (1995).

3 T. B. Pittman, Y. H. Shih, D. V. Strekalov, and A. V. Sergienko, Phys. Rev. A 52, R3429-R3432 (1995).

4 R. Hanbury Brown and R. Q. Twiss, Nature, 178, 1046- 1048 (1956).

5 A. B. Haner and N. R. Isenor, American Journal of Physics, 38, 6, P. 748-751 (1970).

6 Y.-H. Zhai, X.-H. Chen, D. Zhang, and L.-A. Wu, Phys. Rev. A 72, 043805 (2005).

7 R. I. Pfleegor and L. Mandel, Phys. Rev. 159, 1084-1088 (1967); Z. Y. Ou, E. C. Gage, B. E. Magill, and L. Mandel, Optics Communications, 69 (1), 1-5 (1988).

8 A. Gatti, E. Brambilla, M. Bache, and L. A. Lugiato, Phys. Rev. Lett. 93, 093620 (2004); F. Ferri, D. Magatti, A. Gatti, M. Bache, E. Brambilla, and L. A. Lugiato, Phys. Rev. Lett. 94, 183602 (2005).

9 R. S. Bennink, S. J. Bentley, and R.W. Boyd, Phys. Rev. Lett. 89, 113601 (2002).

10 G. Scarcelli, A. Valencia, and Y. Shih, Phys. Rev. A 70, 051802(R) (2004); M. DAngelo, A. Valencia, M. H. Rubin, and Y. Shih, Phys. Rev.A 72, 013810 (2005).

11 Brian J Smith and M G Raymer, New J.phys. 9, 414 (2007).

12 M. Schwartz, Principles of Electrodynamics, Oxford University Press, 1972.

13 P.S.Epstein and P.Ehrenfest, Proc. Nat. Acad. Sci. 10, 133 (1924).

14 H.F. Meiners, Physics Demonstration Experiments, Volume II, The Ronald Press Co., New York, 1970. 

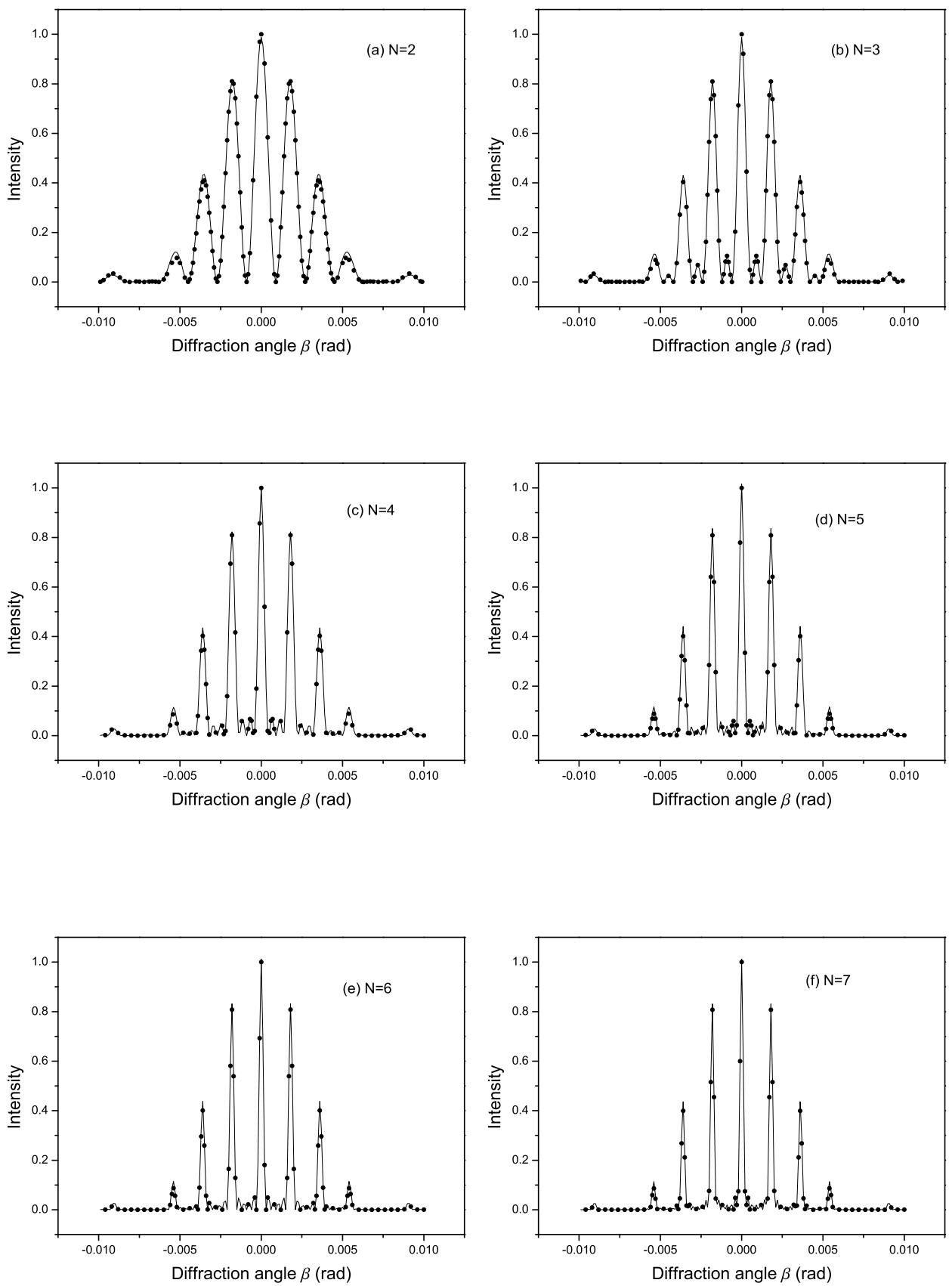

FIG. 4: The diffraction patterns from two, three, four, five, six and seven slits with $a=0.88 \times 10^{-4} \mathrm{~m}, a+d=3.52 \times 10^{-4} \mathrm{~m}, b=4 \times 0.88 \times 10^{-4} \mathrm{~m}, c^{\prime}=0.88 \times 10^{-4} \mathrm{~m}$.

The solid curve is our theoretical calculations and the dot curve is the result of Eq. (48). 


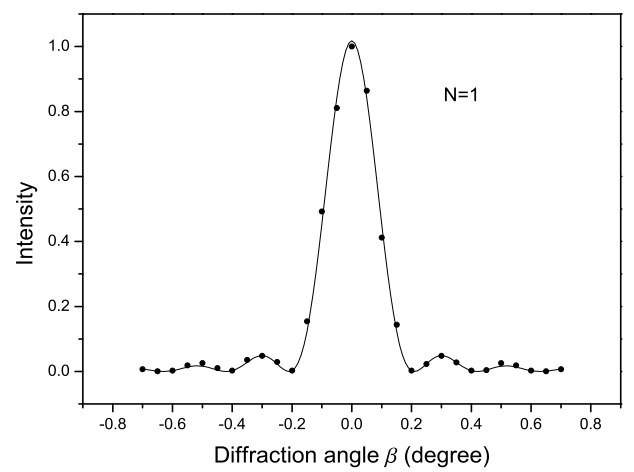

FIG. 5: The diffraction patterns from single slit with $a=1.76 \times 10^{-4} \mathrm{~m}, b=4.0 \times 10^{-4} \mathrm{~m}$ and $c^{\prime}=1.1 \times 10^{-6} \mathrm{~m}$.

The solid curve is our theoretical results and the dot curve is the result of Eq. (48).

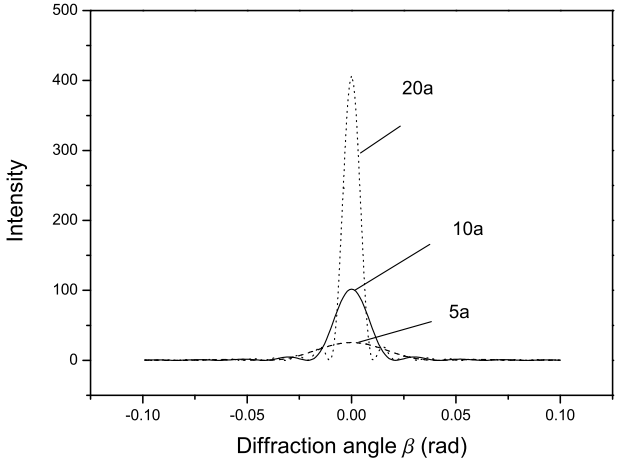

FIG. 6: The diffraction patterns from single slit with $b=4.0 \times 10^{-4} \mathrm{~m}$ and $c^{\prime}=1.1 \times 10^{-6} \mathrm{~m}$. The dash, solid and dot curves correspond to slit width $5 a$, $10 a$ and $20 a\left(a=1.76 \times 10^{-4} \mathrm{~m}\right)$, respectively.

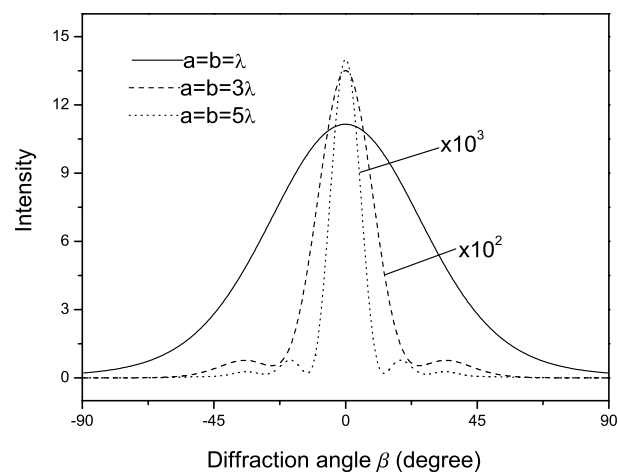

FIG. 7: The diffraction patterns from single slit with $c^{\prime}=1.1 \times 10^{-6} \mathrm{~m}$. The solid, dash and dot curves correspond to $a=b=\lambda, a=b=3 \lambda$ and $a=b=5 \lambda$.

Their real intensity $I$ should be multiplied by $10^{2}$ and $10^{3}$.

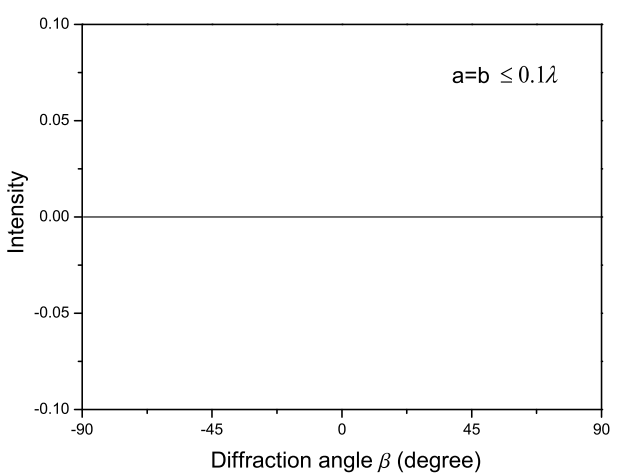

FIG. 8: The diffraction patterns from single slit with $c^{\prime}=1.1 \times 10^{-6} m$ and $a=b \leq 0.1 \lambda$. 


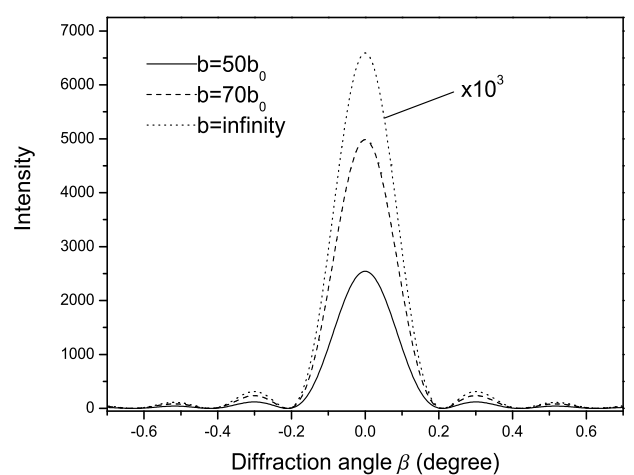

FIG. 9: The diffraction patterns from single slit with $a=1.76 \times 10^{-4} \mathrm{~m}$ and $c^{\prime}=1.1 \times 10^{-6} \mathrm{~m}$. The solid, dash and dot curves curves correspond to slit length $50 b_{0}, 70 b_{0}$ and infinity $\left(b_{0}=4.0 \times 10^{-4} \mathrm{~m}\right)$, respectively. The real intensity $I$ should be multiplied by $10^{3}$.

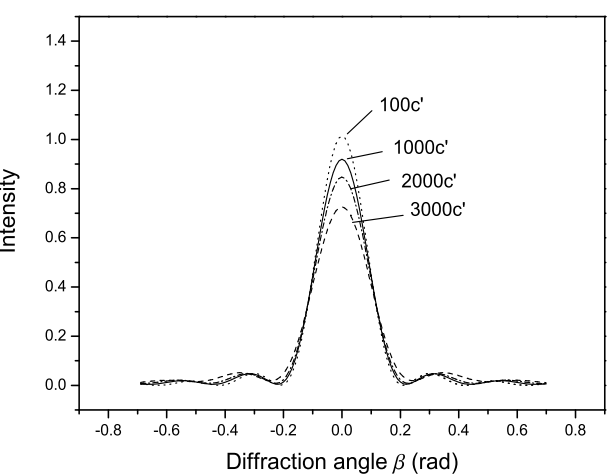

FIG. 10:The diffraction patterns from single slit with $a=1.76 \times 10^{-4} \mathrm{~m}$ and $b=4.0 \times 10^{-4} \mathrm{~m}$. The dot, solid, dash-dot and dash curves correspond to slit thickness $100 c^{\prime}$, $1000 c^{\prime}, 2000 c^{\prime}$ and $3000 c^{\prime}\left(c^{\prime}=1.1 \times 10^{-6} \mathrm{~m}\right)$, respectively.

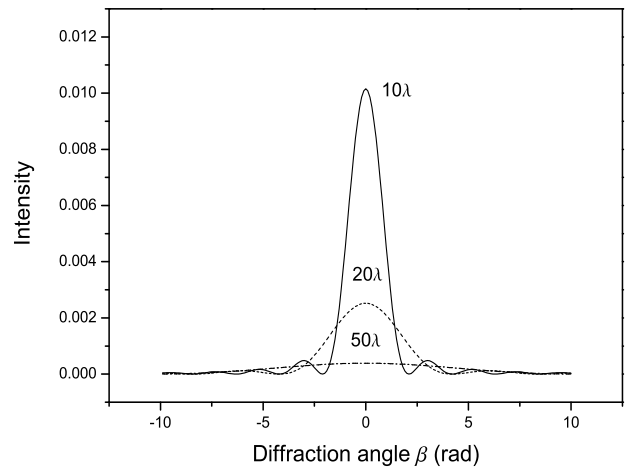

FIG. 11: The diffraction patterns from single slit with $a=1.76 \times 10^{-4} \mathrm{~m}, b=4.0 \times 10^{-4} \mathrm{~m}$ and $c^{\prime}=1.1 \times 10^{-6} \mathrm{~m}$. The solid, dash and dash-dot curves correspond to wave length $10 \lambda, 20 \lambda$ and $50 \lambda$.

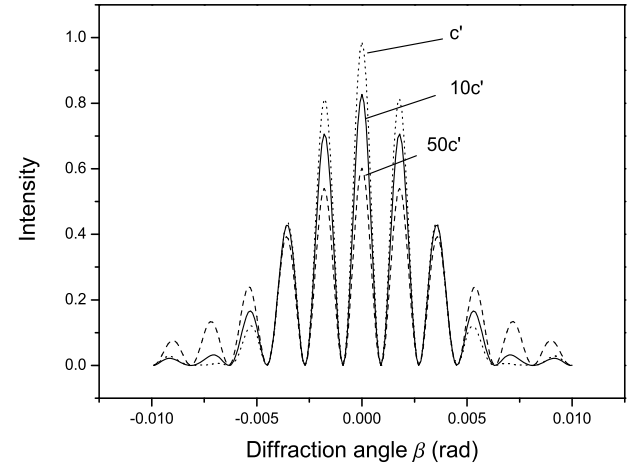

FIG. 12:The diffraction patterns from double slit with $a=0.88 \times 10^{-4} \mathrm{~m}, b=4 \times 0.88 \times 10^{-4} \mathrm{~m}$ and $a+d=3.52 \times 10^{-4} \mathrm{~m}$. The dot, solid and dash curves correspond to slit thickness $c^{\prime}, 10 c^{\prime}$ and $50 c^{\prime}$. 